\title{
Pacific
}

Journal of

Mathematics

\section{THE CHEEGER CONSTANT OF CURVED STRIPS}

DAVID KREJČIIŔíK AND Aldo PRATELLI 


\title{
THE CHEEGER CONSTANT OF CURVED STRIPS
}

\author{
DAVID KREJČİ̌í́K AND Aldo PRATELli
}

\begin{abstract}
We study the Cheeger constant and Cheeger set for domains obtained as strip-like neighborhoods of curves in the plane. If the reference curve is complete and finite (a "curved annulus"), then the strip itself is a Cheeger set and the Cheeger constant equals the inverse of the half-width of the strip. The latter holds true for unbounded strips as well, but there is no Cheeger set. Finally, for strips about noncomplete finite curves, we derive lower and upper bounds to the Cheeger set, which become sharp for infinite curves. The paper is concluded by numerical results for circular sectors.
\end{abstract}

\section{Introduction}

Let $\Omega$ be an open connected set in the plane $\mathbb{R}^{2}$. The Cheeger constant of $\Omega$ is defined as

$$
h(\Omega):=\inf _{S \subseteq \Omega} \frac{P(S)}{|S|},
$$

where the infimum is taken over all sets $S \subseteq \Omega$ of finite perimeter. We use $P(S)$ and $|S|$ to denote the perimeter and the area of $S$, respectively. Any minimizer of (1), if it exists, is called a Cheeger set of $\Omega$ and is denoted by $\mathscr{C}_{\Omega}$.

The problems of existence, uniqueness, and regularity of Cheeger sets have been widely studied in recent years; see, for example, [Kawohl and Fridman 2003; Hebey and Saintier 2006; Saintier 2007; Caselles et al. 2007]. We briefly list and discuss here some of the known general properties.

Theorem 1.1 (general facts). (i) While for a general $\Omega$ neither existence nor uniqueness is guaranteed, there is always some Cheeger set if $\Omega$ is a bounded open set.

(ii) If $\Omega_{1} \subseteq \Omega_{2}$, then $h\left(\Omega_{1}\right) \geq h\left(\Omega_{2}\right)$, but the strict inclusion does not imply the strict inequality.

This work was partially supported by the Czech Ministry of Education, Youth and Sports within the project LC06002, by the Spanish Ministry of Education and Science within the project MTM200803541, and by the ERC within the advanced grant number 226234 and the starting grant number 258685. Krejčiřík acknowledges support from Ikerbasque (Basque Foundation for Science), Spain. MSC2010: 28A75, 49Q20, 35P15, 51M16.

Keywords: Cheeger sets, Cheeger constant, curved strips. 
(iii) The boundary of any Cheeger set $\mathscr{C}_{\Omega}$ intersects the boundary of the set $\Omega$.

(iv) The part of $\partial \mathscr{C}_{\Omega}$ which is inside $\Omega$ is made by arcs of circle, each of which starts and ends touching the boundary of $\Omega$ and is of radius $1 / h(\Omega)$.

(v) A Cheeger set cannot have corners (that is, discontinuities in the tangent vector to the boundary giving rise to an angle smaller than $\pi$ ). In particular, the arcs of circle of $\partial \mathscr{C} \cap \Omega$ must intersect the boundary of $\Omega$ tangentially or in "open corners" (that is, angles bigger than $\pi$ ).

(vi) If there is a Cheeger set, there is a connected Cheeger set.

Concerning property (i), examples of nonexistence or nonuniqueness can be found in [Kawohl and Lachand-Robert 2006], while the existence is immediate by the compactness results for BV functions; see, for example, [Evans and Gariepy 1992; Ambrosio et al. 2000]. Property (ii) is immediate by the definition (1), and examples for the nonstrict inequality can be found in [Kawohl and Lachand-Robert 2006]. Property (iii) comes immediately by a rescaling of $\mathscr{C}$ with a factor bigger than 1 , since this lowers the ratio in (1). Property (iv) comes from a standard variational argument; see, for example, [Kawohl and Fridman 2003, Remark 9]. Property (v) comes directly by noticing that "cutting a corner" of a small length $\varepsilon$ decreases $\left|\mathscr{C}_{\Omega}\right|$ by at most $C \varepsilon^{2}$ and the perimeter by at least $c \varepsilon$. By "corner" we mean a point of the boundary where the tangent vector is discontinuous and makes an angle smaller than $\pi$ (with respect to the internal part of $\Omega$ ). In the case of angles bigger than $\pi$, we talk about "open corners", and they cannot be excluded from $\partial \mathscr{C}$, since, as pointed out in [Kawohl and Lachand-Robert 2006], there are open corners (or "reentrant corners" in their terminology) in an L-shaped set. Finally, property (vi) is immediate because if a Cheeger set has different connected components, each of these components must also be a Cheeger set thanks to the characterization (1).

Apart from the above-mentioned general properties, it is usually a difficult task to find the Cheeger constant or the Cheeger set of a given domain $\Omega$. The situation is simplified when $\Omega$ is a bounded convex set, which is a well-studied situation. In fact, in this case it is known that there is a unique open Cheeger set, which is also convex; see [Alter et al. 2005; Kawohl and Lachand-Robert 2006; Caselles et al. 2007]. Moreover, it is possible to give the following characterization.

Theorem 1.2 [Kawohl and Lachand-Robert 2006]. Let $\Omega$ be a bounded convex subset of $\mathbb{R}^{2}$. For $r \geq 0$, define

$$
\Omega^{r}:=\{x \in \Omega: \operatorname{dist}(x, \partial \Omega)>r\} .
$$

There exists a unique value $r=r^{*}>0$ such that

$$
\left|\Omega^{r}\right|=\pi r^{2} \text {. }
$$


Then $h(\Omega)=1 / r^{*}$ and the Cheeger set of $\Omega$ is the Minkowski sum $\mathscr{C}_{\Omega}=\Omega^{r^{*}}+B_{r^{*}}$, with $B_{r^{*}}$ denoting the disc of radius $r^{*}$.

This theorem can be used to find explicitly $h(\Omega)$ and $\mathscr{C}_{\Omega}$ in some cases (for example for discs, rectangles and triangles). In particular, the Cheeger sets of rectangles and triangles are obtained by suitably "cutting the corners". Furthermore, it provides a constructive algorithm for the determination of the Cheeger constant and Cheeger set for general convex domains; in particular for convex polygons.

Unfortunately, there is no such constructive method for nonconvex domains. Only one particular case seems to be explicitly known in the literature, namely the annulus, for which it is known that $\mathfrak{C}_{\Omega}=\Omega$. In general, while a trivial strategy to find upper estimates for $h(\Omega)$ is to choose a suitable "test domain" $S$ in (1), it is less clear how to obtain lower estimates. One possibility is given by the following result concerning "test vector fields".

Theorem 1.3 [Grieser 2006]. Let $V: \Omega \rightarrow \mathbb{R}^{2}$ be a smooth vector field on $\Omega, h \in \mathbb{R}$, and assume that the pointwise inequalities $|V| \leq 1$ and $\operatorname{div} V \geq h$ hold in $\Omega$. Then $h(\Omega) \geq h$.

An example of the applicability of this criterion is the above-mentioned result for the annulus, which can be obtained by employing the vector field of [Bellettini et al. 2002, Section 11, Example 4] (see also Remark 2.6 below, where the corresponding vector field can be found explicitly). However, for a general set $\Omega$ it is not easy at all to find a vector field producing nontrivial lower bounds by this criterion.

The purpose of this paper is to introduce a class of nonconvex planar domains for which the Cheeger constant and the Cheeger set can be determined explicitly, namely the curved strips. This class of sets has been intensively studied in the last two decades as an effective configuration space for curved quantum waveguides (see [Duclos and Exner 1995; Krejčiřík and Kříž 2005] and the references therein).

More precisely, we call a tubular neighborhood of a curve without boundary in the plane a "curved strip". There are then few possibilities: a "curved annulus", a "finite curved strip", an "infinite curved strip", or a "semi-infinite curved strip"; see Figure 1 (we leave the formal definitions to Section 1).

Our main results, Theorems 3.1 and 3.2, describe the situation in all of these cases. In particular, for a curved annulus the situation is analogous to the standard annulus, that is, the strip itself is the unique Cheeger set and the Cheeger constant only depends on the width of the strip, irrespectively of the curvature of the curve. More precisely, the Cheeger constant is the inverse of the half-width (Theorem 3.1, part (i)). For an infinite or a semi-infinite curved strip, again the Cheeger constant equals the inverse of the half-width of the strip, but there is no Cheeger set (Theorem 3.1, part (ii)). Finally, for a finite curved strip, the situation is analogous to the standard rectangle, that is, there exists a Cheeger set, which 


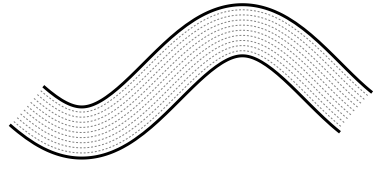

infinite curved strip

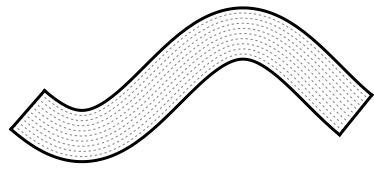

finite curved strip

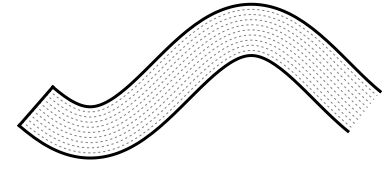

semi-infinite curved strip

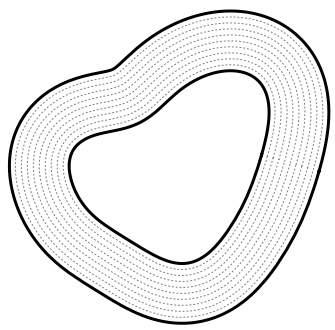

curved annulus

Figure 1. The four possible types of strips.

is not the whole strip because of the corners, and the Cheeger constant is strictly bigger than the inverse of the half-width. Moreover, in this last case we can also give a (sharp) upper and a lower bound, which depend only on the width and length of the strip (Theorem 3.2).

We conclude this introductory section with a couple of comments. First of all, it should mentioned that in the study of the Cheeger problem an important role is played by those sets $\Omega$ which are Cheeger sets of themselves. This is what happens in many situations, such as the discs, the annuli, and, as we show in the present paper, the "curved annuli". Those sets are called calibrable and are intensively studied in the image processing literature; see, for instance, [Bellettini et al. 2002].

A second remark has to be made on the connection between the Cheeger constant and the eigenvalue problems. In fact, the Cheeger inequality tells us that

$$
\lambda_{p}(\Omega) \geq\left(\frac{h(\Omega)}{p}\right)^{p}
$$

for any $p \in(1, \infty)$, where $\lambda_{p}(\Omega)$ is the first eigenvalue of the $p$-Laplacian. Moreover, as shown in [Kawohl and Fridman 2003], $h(\Omega)=\lim _{p \searrow 1} \lambda_{p}(\Omega)$. In this regard, it is interesting to notice one property of the curved strips. It is well known that the first eigenvalue of the Dirichlet Laplacian (or, more generally, the infimum of the Rayleigh quotient, in the case of unbounded strips for which there might be no eigenvalues) for a curved strip strongly depends on its curvature; see, for instance, [Duclos and Exner 1995; Exner et al. 2004; Krejčiřík and Kř́̌́z 2005]. On the other hand, the Cheeger constant is much less sensitive, because, as we will 
show, for infinite and semi-infinite curve strips, as well as for curved annuli, the Cheeger constant depends not on the curvature of the strip, but on its width.

The geometrical setting. In this section we set the notations for the geometrical situation that we will consider throughout the paper. Let $\Gamma$ be a $C^{2}$, connected curve in $\mathbb{R}^{2}$ (that is, the homeomorphic image of $(0,1)$ or $\mathbb{S}^{1}$ under a $C^{2}$ function), and let us denote by $|\Gamma|=\int_{\Gamma} d q$ its length, $d q$ being the arclength element of $\Gamma$. Additionally, let $N: \Gamma \rightarrow \mathbb{R}^{2}$ be a $\mathrm{C}^{1}$ vector field giving the normal vector in the points of $\Gamma$, and let $\kappa: \Gamma \rightarrow \mathbb{R}$ be the associated curvature (notice that the sign of $\kappa$ depends on the choice of the orientation of $N$ ). We recall that to define $\kappa$ it is enough to take a unit-speed parametrization $\gamma$ of $\Gamma$, and hence it is

$$
\kappa(q)=\ddot{\gamma}\left(\gamma^{-1}(q)\right) \cdot N(q),
$$

where the dot denotes the standard scalar product in $\mathbb{R}^{2}$. We now introduce a mapping $\mathscr{L}$ from $\Gamma \times \mathbb{R}$ to $\mathbb{R}^{2}$,

$$
\mathscr{L}(q, t):=q+t N(q),
$$

and we introduce the set

$$
\Omega_{\Gamma, a}:=\mathscr{L}(\Gamma \times(-a, a)),
$$

for any positive $a$. We are interested in sets $\Omega_{\Gamma, a}$ that are non-self-intersecting tubular neighborhoods of $\Gamma$. More precisely, we will always assume that

$$
\mathscr{L} \text { is injective in } \Gamma \times[-a, a],
$$

hence the set is as in Figure 2. Using the expression for the bilinear form

$$
d \mathscr{L}^{2}=(1-\kappa(q) t)^{2} d q^{2}+d t^{2}
$$

that follows from (4), we can easily see that, by the inverse function theorem, the assumption (5) forces $a$ to be small compared to the curvature. More precisely, (5) implies that $|\kappa(q)| a \leq 1$ for any $q \in \Gamma$, the boundary of $\Omega_{\Gamma, a}$ is $\mathrm{C}^{1,1}$, and $\mathscr{L}$ is in fact a $C^{1}$ diffeomorphism between $\Gamma \times(-a, a)$ and $\Omega_{\Gamma, a}$.

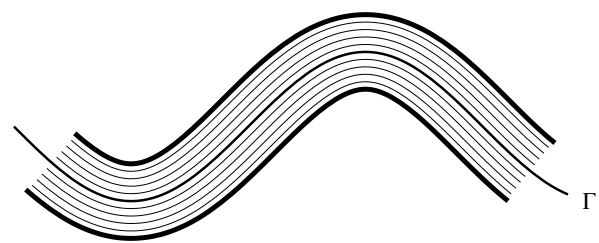

Figure 2. The geometry of a curved strip $\Omega_{\Gamma, a}$ and the corresponding curve $\Gamma$; the parallel lines correspond to the curves $s \mapsto \mathscr{L}(s, t)$ with fixed $t \in(-a, a)$. 
Summing up, under the hypothesis (5) $\Omega_{\Gamma, a}$ has the geometrical meaning of an open non-self-intersecting strip contained between the parallel curves

$$
q \mapsto q \pm a N(q),
$$

with $q \in \Gamma$, and it can be identified with the Riemannian manifold $\Gamma \times(-a, a)$ equipped with the metric (6).

In this paper, we will call any set $\Omega_{\Gamma, a}$ satisfying the assumption (5) a curved strip. Notice that when $\Gamma$ is contained in a line, $\Omega$ reduces to a rectangle. But the most interesting situation is when $\Gamma$ has a more complicated geometry, since then the associated set is not convex, hence not covered by the preceding known results for the Cheeger problem. It is easy to characterize the four possible situations occurring for a curved strip, to each of which we will associate a name to fix the ideas. The four kinds of strips are shown in Figure 1. First of all, if the curve $\Gamma$ is not finite, it may be either infinite or semi-infinite (that is, not finite but complete, or not finite and not complete, respectively). We will call the corresponding sets $\Omega_{\Gamma, a}$ an infinite curved strip and a semi-infinite curved strip. On the other hand, if the curve is finite, then it can be either compact or not compact (homeomorphic to a circle or to an open segment, respectively). In the first case, we will speak about a curved annulus, the annulus corresponding to the case when $\Gamma$ is exactly a circle, and in the other case about a finite curved strip.

\section{The main geometrical results}

In this section we give some general technical properties, which will be used later to show our main results. We can easily obtain an upper bound for the curved strips. In the next result, for a curve $\Gamma$ which is not finite, we consider a unit-speed parametrization $\gamma:(0,+\infty) \rightarrow \mathbb{R}^{2}$ (respectively, $\left.\gamma:(-\infty,+\infty) \rightarrow \mathbb{R}^{2}\right)$ if the strip is semi-infinite (respectively, infinite). We will denote by $\Gamma_{L}$ the subset of $\Gamma$ given by $\gamma(0, L)$ or $\gamma(-L, L)$ for the semi-infinite or infinite case, respectively.

Lemma 2.1 (upper bound). Let $\Gamma$ be infinite or compact (that is, $\Omega_{\Gamma, a}$ is a semiinfinite or infinite curved strip, or a curved annulus). Then

$$
h\left(\Omega_{\Gamma, a}\right) \leq \frac{1}{a} .
$$

In particular, if $\Omega_{\Gamma, a}$ is a curved annulus,

$$
\frac{P\left(\Omega_{\Gamma, a}\right)}{\left|\Omega_{\Gamma, a}\right|}=\frac{1}{a},
$$

while if $\Omega_{\Gamma, a}$ is a semi-infinite or infinite curved strip,

$$
\frac{P\left(\Omega_{\Gamma_{L}, a}\right)}{\left|\Omega_{\Gamma_{L}, a}\right|} \underset{L \rightarrow \infty}{\longrightarrow} \frac{1}{a} .
$$


Proof. If $\Omega_{\Gamma, a}$ is a curved annulus, then we take the whole $S=\Omega_{\Gamma, a}$ as a test domain in (1). Recalling (6), we then have

$$
\frac{P(S)}{|S|}=\frac{\int_{\Gamma}(1+\kappa(q) a) d q+\int_{\Gamma}(1-\kappa(q) a) d q}{\int_{\Gamma} \int_{-a}^{a}(1-\kappa(q) t) d t d q}=\frac{2|\Gamma|}{2 a|\Gamma|}=\frac{1}{a} .
$$

Notice that, by the symmetry of the set $S$, the curvature term cancels both in the numerator and in the denominator.

On the other hand, if $\Gamma$ is not finite, then the whole strip is not admissible because it has both infinite area and perimeter. However, for any $L>0$, we can consider the finite curved strip $S=\Omega_{\Gamma_{L}, a}$, which is of course contained in $\Omega_{\Gamma, a}$. Therefore, one can easily evaluate

$$
\begin{aligned}
\frac{P(S)}{|S|} & =\frac{4 a+\int_{\Gamma_{L}}(1+\kappa(q) a) d q+\int_{\Gamma_{L}}(1-\kappa(q) a) d q}{\int_{\Gamma_{L}} \int_{-a}^{a}(1-\kappa(q) t) d t d q} \\
& =\frac{4 a+2\left|\Gamma_{L}\right|}{2 a\left|\Gamma_{L}\right|} \underset{L \rightarrow \infty}{\longrightarrow} \frac{1}{a} .
\end{aligned}
$$

In the formula for the perimeter, notice the term $4 a$ corresponding to the two "vertical" parts of $\partial S$ at the start and at the end. Thanks to the definition (1), the two above estimates give the thesis.

The lower bound is much more complicated to obtain. To find it, we introduce an operation that, in a sense, fills in the "holes" and the "bays" in the test domains $S$. More precisely, let us take an open set $S \subseteq \Omega_{\Gamma, a}$, and define the set $\Gamma_{S}$ as

$$
\Gamma_{S}:=\{q \in \Gamma: \mathscr{L}(\{q\} \times(-a, a)) \cap S \neq \varnothing\},
$$

and the functions $f_{ \pm}: \Gamma_{S} \rightarrow[-a, a]$ as

$$
f_{-}(q):=\inf \{t \in(-a, a):(q, t) \in S\}, \quad f_{+}(q):=\sup \{t \in(-a, a):(q, t) \in S\} .
$$

Therefore, $S$ is contained between the two graphs of $f_{+}$and $f_{-}$. Notice now that, if $S$ is connected, then of course so is $\Gamma_{S}$. In particular, there are two possibilities: either $\Gamma_{S}$ is a subinterval of $\Gamma$ and, in this case, we call $q_{l}$ and $q_{r}$ its extremes, or $\Gamma_{S}$ is a closed curve. Observe that if $\Gamma$ is not compact (that is, always except when $\Omega_{\Gamma, a}$ is a curved annulus), then $\Gamma_{S}$ must necessarily be a subinterval of $\Gamma$; on the other hand, if $\Omega_{\Gamma, a}$ is a curved annulus, then both the situations - that $\Gamma_{S}$ is a subinterval of $\Gamma$, and that $\Gamma_{S}$ is a closed curve - are possible, and, in particular, $\Gamma_{S}$ is a closed curve if and only if $\Gamma_{S}=\Gamma$.

Definition 2.2. Let $S$ be an open subset of $\Omega_{\Gamma, a}$ with finite perimeter, and let $\Gamma_{S}$ and $f_{ \pm}$be defined as above. We define

$$
S^{*}:=\left\{\mathscr{L}(q, t) \in \Omega_{\Gamma, a}: q \in \Gamma_{S}, f_{-}(q)<t<f_{+}(q)\right\} .
$$


We can now show the main property of the set $S^{*}$, which will be fundamental for our purposes.

Lemma 2.3 (area and perimeter of $S^{*}$ ). Let $S$ be an open, bounded, and connected subset of $\Omega$ of finite perimeter. Then

$$
\left|S^{*}\right| \geq|S|, \quad P\left(S^{*}\right) \leq P(S),
$$

and $f_{ \pm} \in B V\left(\Gamma_{S}\right)$. Moreover, calling $f_{ \pm}^{\prime} d q$ the absolute continuous part of $D f_{ \pm}$, and $D_{s} f_{ \pm}$its singular part, we have the validity of the formula

$$
\begin{aligned}
P\left(S^{*}\right)= & \int_{\Gamma_{S}} \sqrt{\left(1-\kappa(q) f_{+}(q)\right)^{2}+f_{+}^{\prime}(q)^{2}} d q \\
& +\int_{\Gamma_{S}} \sqrt{\left(1-\kappa(q) f_{-}(q)\right)^{2}+f_{-}^{\prime}(q)^{2}} d q+\left|D_{s} f_{+}\right|\left(\Gamma_{S}\right) \\
& \quad+\left|D_{s} f_{-}\right|\left(\Gamma_{S}\right)+\left(f_{+}\left(q_{l}\right)-f_{-}\left(q_{l}\right)+\left(f_{+}\left(q_{r}\right)-f_{-}\left(q_{r}\right)\right),\right.
\end{aligned}
$$

where if $\Gamma_{S}$ is a subinterval of $\Gamma$, we denote by $q_{l}$ and $q_{r}$ its extremes, and if $\Gamma_{S}$ is compact, the term $\left(f_{+}\left(q_{l}\right)-f_{-}\left(q_{l}\right)\right)+\left(f_{+}\left(q_{r}\right)-f_{-}\left(q_{r}\right)\right)$ has to be intended as 0 .

Proof. First of all, the fact that $\left|S^{*}\right| \geq|S|$ is obvious, since by definition $S^{*} \supseteq S$. Concerning the inequality for the perimeter, we start by noticing that, by standard arguments, it is admissible to assume that $S$ is smooth. In fact, by the compactness theorem for BV functions [Ambrosio et al. 2000], we can take a sequence $S_{j}$ of smooth sets converging in the $L^{1}$ sense to $S$ in such a way that $P\left(S_{j}\right) \rightarrow P(S)$. By definition, the corresponding sets $S_{j}^{*}$ converge to $S^{*}$, and by the lower semicontinuity of the perimeter this yields $P\left(S^{*}\right) \leq \liminf P\left(S_{j}^{*}\right)$. As an immediate consequence, once we establish the validity of this lemma for smooth sets, it will directly follow also in full generality.

The inequality $P\left(S^{*}\right) \leq P(S)$ for smooth sets is very easy to guess, but a bit boring to prove. For simplicity, we will divide the proof into several steps.

Step I. Nonintersecting curves cannot pass "from above to below". In this first step, we underline the following very easy topological fact.

Claim. Let $\gamma_{1}, \gamma_{2} \subseteq \mathbb{R}^{2}$ be continuous, nonintersecting plane curves such that $\min \pi_{1} \gamma_{1}=\min \pi_{1} \gamma_{2}=q_{0} \in \mathbb{R}$, where $\pi_{1}: \mathbb{R}^{2} \rightarrow \mathbb{R}$ denotes the first projection. If $\max \left\{t:\left(q_{0}, t\right) \in \gamma_{1}\right\}=t_{1}$ and $\max \left\{t:\left(q_{0}, t\right) \in \gamma_{2}\right\}=t_{2}$, with $t_{1}>t_{2}$, then $\max \left\{t:(q, t) \in \gamma_{1}\right\}>\max \left\{t:(q, t) \in \gamma_{2}\right\}$ for all $q \in \pi_{1} \gamma_{1} \cap \pi_{1} \gamma_{2}$.

The meaning of this claim is very simple: if one has two continuous and nonintersecting curves in the plane, and the least abscissa of points in the two curves coincide (otherwise, it is obvious that the claim is false), then the curve which starts above always remains above. 

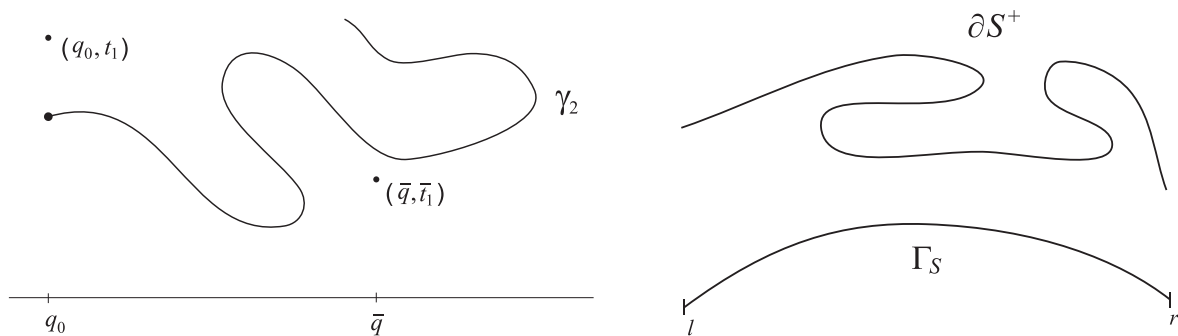

Figure 3. Left: the situation of Step I in the proof of Lemma 2.3.

Right: a possible $\partial S^{+}$in Step III.

To show the validity of the claim, suppose it is not true, and let $\bar{q} \in \pi_{1} \gamma_{1} \cap \pi_{1} \gamma_{2}$ be a point for which

$$
\bar{t}_{1}:=\max \left\{t:(\bar{q}, t) \in \gamma_{1}\right\}<\max \left\{t:(\bar{q}, t) \in \gamma_{2}\right\}=: \bar{t}_{2} .
$$

Because the curves do not intersect the equality cannot hold true. Figure 3, left, shows the situation.

The curve $\gamma_{1}$, then, is contained by definition in

$$
A:=\left\{(q, t) \in \mathbb{R}^{2} \backslash \gamma_{2}: q \geq q_{0},\right\} \backslash\left\{(\bar{q}, t) \in \mathbb{R}^{2}: t>\bar{t}_{1}\right\} .
$$

This is a contradiction with the continuity of the curve $\gamma_{1}$, since the points $\left(q_{0}, t_{1}\right)$ and $\left(\bar{q}, \bar{t}_{1}\right)$ are in $\gamma_{1}$ but belong to two distinct connected components of $A$. Therefore, the Claim is proved.

Step II. First properties and some definitions. We can immediately observe some simple properties of $\partial S$ and give some related definitions. First of all, since $S$ is smooth, $\partial S$ is the union of finitely many closed curves $\gamma_{i}, 1 \leq i \leq N$. Exactly one of them, say $\gamma_{1}$, encloses all of $S$.

Let us then consider the two cases ( $\Gamma_{S}$ is either compact or not) separately. If $\Gamma_{S}$ is not compact and is thus an interval $\left(q_{l}, q_{r}\right)$, then it is immediate to observe that for every $q \in \Gamma_{S}$ the points $\mathscr{L}\left(q, f_{ \pm}(q)\right)$ belong to $\gamma_{1}$. Let us then call $\partial S^{+}$the part of $\gamma_{1}$ starting from $\mathscr{L}\left(l, f_{+}(l)\right)$, ending at $\mathscr{L}\left(r, f_{+}(r)\right)$ and containing $\mathscr{L}\left(q, f_{+}(q)\right)$ for every $q \in \Gamma_{S}$; similarly, we denote by $\partial S^{-}$the part of $\gamma_{1}$ starting from $\mathscr{L}\left(r, f_{-}(r)\right)$, ending at $\mathscr{L}\left(l, f_{-}(l)\right)$, and containing $\mathscr{L}\left(q, f_{-}(q)\right)$ for every $q \in \Gamma_{S}$. An easy geometric argument ensures that $\partial S^{+}$and $\partial S^{-}$are well defined and do not intersect each other. We then obtain

$$
\partial S \supseteq \gamma_{1}=\partial S^{+} \cup \partial S^{r} \cup \partial S^{-} \cup \partial S^{l},
$$

being $\partial S^{r}$ (respectively $\left.\partial S^{l}\right)$ the part of $\gamma \backslash\left(\partial S^{+} \cup \partial S^{-}\right)$connecting $\mathscr{L}\left(r, f_{+}(r)\right)$ and $\mathscr{L}\left(r, f_{-}(r)\right)$ (respectively $\mathscr{L}\left(l, f_{-}(l)\right)$ and $\left.\mathscr{L}\left(l, f_{+}(l)\right)\right)$. 
Consider now the case when $\Gamma_{S}$ is compact $\left(\Gamma_{S}=\Gamma\right)$. In this case, we can directly call $\partial S^{+}=\gamma_{1}$, and again it is easy to observe that for every $q \in \Gamma$ one has $\mathscr{L}\left(q, f_{+}(q)\right) \in \partial S^{+}$. On the other hand, all the points $\mathscr{L}\left(q, f_{-}(q)\right)$ belong to the same connected component of $\partial S$ different from $\gamma_{1}$, say $\gamma_{2}$. We then call $\partial S^{-}=\gamma_{2}$ and $\partial S^{r}=\partial S^{l}=\varnothing$, so that also in this case (10) holds true.

We conclude this step noticing that, for the set $S^{*}$, the inclusion (10) is in fact an equality by construction.

Step III. The "upper boundary" is well-ordered. We show that the curve $\partial S^{+}$ reaches all the points $\mathscr{L}\left(q, f_{+}(q)\right)$ in the "correct order". This means that, if we parametrize $\partial S^{+}$as $\gamma([0,1])$ with $\gamma(0)=\mathscr{L}\left(l, f_{+}(l)\right)$ and $\gamma(1)=\mathscr{L}\left(r, f_{+}(r)\right)$, then

(11) If $\gamma\left(\sigma_{1}\right)=\mathscr{L}\left(q_{1}, f_{+}\left(q_{1}\right)\right), \gamma\left(\sigma_{2}\right)=\mathscr{L}\left(q_{2}, f_{+}\left(q_{2}\right)\right)$, then $\sigma_{1}<\sigma_{2} \Longleftrightarrow q_{1}<q_{2}$.

Notice that this fact is not trivial, since the curve $2 S^{+}$does not have to be a graph on $\Gamma_{S}$, and, therefore, it can sometimes move to the left, as in Figure 3, right. However, the figure itself suggests that the points $\left(q, f_{+}(q)\right)$ are in any case reached "from left to right". Let us now show (11). To do so, suppose by contradiction that it is not true. Hence, there exist $\sigma_{1}, \sigma_{2}, q_{1}$, and $q_{2}$ such that $\gamma\left(\sigma_{i}\right)=\mathscr{L}\left(q_{i}, f_{+}\left(q_{i}\right)\right)$ for $i=1,2$, but one has $\sigma_{1}>\sigma_{2}$ and $q_{1}<q_{2}$. We can then give the following definitions, $\pi$ being the projection from $\Omega$ to $\Gamma$.

$$
\begin{aligned}
& \sigma_{3}=\min \left\{\sigma \in\left(\sigma_{1}, 1\right): \pi(\gamma(\sigma))=q_{2}\right\} \\
& q^{*}=\min \left\{\pi(\gamma(\sigma)): \sigma \in\left(\sigma_{1}, \sigma_{3}\right)\right\}, \\
& \sigma_{0}=\max \left\{\sigma \in\left(0, \sigma_{2}\right): \pi(\gamma(\sigma))=q^{*}\right\} .
\end{aligned}
$$

By construction one has $0<\sigma_{0}<\sigma_{2}<\sigma_{1}<\sigma_{3}<1$, as well as $q^{*} \leq q_{1}<q_{2}$. Now consider the two curves $\gamma_{1}=\mathscr{L}^{-1}\left(\gamma_{\mid\left[\sigma_{0}, \sigma_{2}\right]}\right)$ and $\gamma_{2}=\mathscr{L}^{-1}\left(\gamma_{\mid\left[\sigma_{1}, \sigma_{3}\right]}\right)$, which are continuous and nonintersecting. Moreover, $\min \pi_{1} \gamma_{1}=\min \pi_{1} \gamma_{2}=q^{*}$, so we can apply Step I to derive that $\gamma_{1}$ is either "always above" or "always below" $\gamma_{2}$, in the sense of the Claim. By checking $q=q_{1}$, we observe that $\gamma_{1}$ is below $\gamma_{2}$, since $\max \left\{\sigma:\left(q_{1}, \sigma\right) \in \gamma_{2}\right\}=f_{+}\left(q_{1}\right)$ is greater than $\max \left\{\sigma:\left(q_{1}, \sigma\right) \in \gamma_{1}\right\}$, by definition of $f_{+}$. On the other hand, by checking $q=q_{2}$, the very same reasoning shows that $\gamma_{1}$ is above $\gamma_{2}$, being $\max \left\{\sigma:\left(q_{2}, \sigma\right) \in \gamma_{1}\right\}=f_{+}\left(q_{2}\right)$. The contradiction shows the validity of (11).

Step IV. The functions $f_{ \pm}$are in $B V\left(\Gamma_{S}\right)$. Let us fix an arbitrary $N \in \mathbb{N}$, and an arbitrary sequence $l=q_{0}<q_{1}<\cdots<q_{N}<q_{N+1}=r$ in $\Gamma_{S}$. We claim that

$$
\sum_{i=0}^{N}\left|f_{+}\left(q_{i}\right)-f_{+}\left(q_{i+1}\right)\right| \leq \mathscr{H}^{1}\left(\partial S^{+}\right),
$$


$\mathscr{H}^{1}$ being Hausdorff measure of dimension one (length). Notice that this inequality would show that $f_{+} \in B V\left(\Gamma_{S}\right)$, since $S$ is of finite perimeter.

To show the estimate, let us call $\gamma_{i}$ the part of the curve $\partial S^{+}$which connects $\mathscr{L}\left(q_{i}, f_{+}\left(q_{i}\right)\right)$ with $\mathscr{L}\left(q_{i+1}, f_{+}\left(q_{i+1}\right)\right)$. By the preceding steps, we know that $\partial S^{+}$ consists of the disjoint union of the curves $\gamma_{i}$, so that

$$
\mathscr{H}^{1}\left(\partial S^{+}\right)=\sum_{i=0}^{N} \mathscr{H}^{1}\left(\gamma_{i}\right) .
$$

Hence (12) will follow because for any $i=0, \ldots, N$, one has

$$
\mathscr{H}^{1}\left(\gamma_{i}\right) \geq\left|\mathscr{L}\left(q_{i}, f_{+}\left(q_{i}\right)\right)-\mathscr{L}\left(q_{i+1}, f_{+}\left(q_{i+1}\right)\right)\right|>\left|f_{+}\left(q_{i}\right)-f_{+}\left(q_{i+1}\right)\right| .
$$

The first inequality is trivial, since it just says that the length of the curve $\gamma_{i}$ is greater than the distance of its extreme points. Concerning the strict inequality let us instead use the following notation for brevity.

$$
\begin{aligned}
& P:=\mathscr{L}\left(q_{i}, f_{+}\left(q_{i}\right)\right), \quad Q:=\mathscr{L}\left(q_{i+1}, f_{+}\left(q_{i+1}\right)\right), \quad Q^{\prime}:=\mathscr{L}\left(q_{i}, f_{+}\left(q_{i+1}\right)\right), \\
& S^{\prime}:=\mathscr{L}\left(q_{i}, 0\right), \quad S:=\mathscr{L}\left(q_{i+1}, 0\right) .
\end{aligned}
$$

Hence, assuming that $f_{+}\left(q_{i}\right) \geq f_{+}\left(q_{i+1}\right) \geq 0$ (it is then trivial to modify the argument to cover the other cases), one has

$$
\overline{P Q^{\prime}}+\overline{Q^{\prime} S^{\prime}}=\overline{P S^{\prime}}<\overline{P S}<\overline{P Q}+\overline{Q S}=\overline{P Q}+\overline{Q^{\prime} S^{\prime}},
$$

where the first inequality is due to the fact that, by definition, $S^{\prime}$ is the closest point to $P$ inside $\Gamma$. The inequality above says that $\overline{P Q^{\prime}}<\overline{P Q}$, which is precisely the missing inequality in (13). As explained above, this implies the validity of (12), hence the fact that $f_{+} \in B V\left(\Gamma_{S}\right)$.

Of course, the very same argument shows that $f_{-} \in B V\left(\Gamma_{S}\right)$.

Step $V$. One has $\mathscr{H}^{1}\left(\partial S^{+}\right) \geq \mathscr{H}^{1}\left(\partial S^{*+}\right)$. Let us define $\left\{q_{i}, i \in \mathbb{N}\right\} \subseteq \Gamma_{S}$ the jump points of $f_{+}$, which are countably many since $f_{+} \in B V\left(\Gamma_{S}\right)$. For any $i$, set

$$
f_{+}^{l}\left(q_{i}\right)=\lim _{q \uparrow q_{i}} f_{+}(q), \quad f_{+}^{r}\left(q_{i}\right)=\lim _{q \downarrow q_{i}} f_{+}(q) .
$$

Since $f_{+} \in B V\left(\Gamma_{S}\right)$, these two limits exist and correspond to the lim inf and the $\lim$ sup of $f_{+}$for $q \rightarrow q_{i}$. In particular, one has that

$$
\partial\left(S^{*+}\right)=\left\{\mathscr{L}\left(q, f_{+}(q)\right): q \in \Gamma_{S}\right\} \cup \bigcup_{i \in \mathbb{N}} J_{i},
$$

where $J_{i}$ is the segment joining $\mathscr{L}\left(q_{i}, f_{+}^{l}\left(q_{i}\right)\right)$ and $\mathscr{L}\left(q_{i}, f_{+}^{r}\left(q_{i}\right)\right)$. Let us now fix $\varepsilon>0$, so that there exists $N \in \mathbb{N}$ such that

$$
\sum_{i>N}\left|J_{i}\right|<\varepsilon .
$$


We can assume that the points $q_{i}$ are ordered so that $l<q_{1}<\cdots<q_{n}<r$. We can now pick, for any $1 \leq i \leq N$, two points $q_{i}^{l}<q_{i}<q_{i}^{r}$ in $\Gamma_{S}$ so that

- the different intervals $\left(q_{i}^{l}, q_{i}^{r}\right)$ are disjoint,

- $\left|f_{+}\left(q_{i}^{l}\right)-f_{+}^{l}\left(q_{i}\right)\right|+\left|f_{+}\left(q_{i}^{r}\right)-f_{+}^{r}\left(q_{i}\right)\right| \leq \frac{\varepsilon}{N}$ for any $i$, and

- $\mathscr{H}^{1}\left(\left(\partial S^{*+}\right) \cap \mathscr{L}\left(\left(q_{i}^{l}, q_{i}^{r}\right) \times(-a, a)\right)\right) \leq\left|J_{i}\right|+\frac{\varepsilon}{N}=\left|f_{+}^{l}\left(q_{i}\right)-f_{+}^{r}\left(q_{i}\right)\right|+\frac{\varepsilon}{N}$.

We now consider the "bad" intervals $B_{i}=\left(q_{i}^{l}, q_{i}^{r}\right)$, where there are high jumps, and the "good" intervals $G_{i}=\left(q_{i}^{r}, q_{i+1}^{l}\right)$, where there are not. Define also $G_{0}=\left(l, q_{1}^{l}\right)$, while $G_{N}=\left(q_{N}^{r}, r\right)$. Therefore, we have decomposed $\Gamma_{S}=\cup_{i \leq N} B_{i} \cup G_{i}$. For any good interval $G_{i}$, one has

$$
\partial S^{*+} \cap \mathscr{L}\left(G_{i} \times(-a, a)\right)=\left\{\mathscr{L}\left(q, f_{+}(q)\right): q \in G_{i}\right\} \cup \bigcup_{j \in \mathbb{N}} \widetilde{J}_{i, j},
$$

where $\widetilde{J}_{i, j}$ are the jumps of $f_{+}$contained in the interval $G_{i}$. Of course all the jumps $\widetilde{J}_{i, j}$, varying $0 \leq i \leq N$ and $j \in \mathbb{N}$, correspond to different jumps $J_{i}$ for $i>N$. For any bad interval $B_{i}$, moreover, call $\gamma_{i}$ the part of the curve $2 S^{+}$from $\mathscr{L}\left(q_{i}^{l}, f_{+}\left(q_{i}^{l}\right)\right)$ to $\mathscr{L}\left(q_{i}^{r}, f_{+}\left(q_{i}^{r}\right)\right)$. Thanks to Step III, all the curves $\gamma_{i}$ are disjoint, and, in particular, $\mathscr{L}\left(q, f_{+}(q)\right)$ belongs to $\gamma_{i}$ if and only if $q \in B_{i}$. Since we know that $\mathscr{L}\left(q, f_{+}(q)\right) \in \partial S^{+}$for all $q \in \Gamma_{S}$, this implies that

$$
\mathscr{H}^{1}\left(\partial S^{+}\right) \geq \mathscr{H}^{1}\left(\left\{\mathscr{L}\left(q, f_{+}(q)\right): q \in \bigcup_{i=0}^{N} G_{i}\right\}\right)+\sum_{i=1}^{N} \mathscr{H}^{1}\left(\gamma_{i}\right) .
$$

Notice also that, as shown with (13) in Step IV, we have that for each $1 \leq i \leq N$

$$
\mathscr{H}^{1}\left(\gamma_{i}\right)>\left|f_{+}\left(q_{i}^{l}\right)-f_{+}\left(q_{i}^{r}\right)\right| .
$$

Finally, using all the properties listed above, we conclude that $\mathscr{H}^{1}\left(\partial S^{*+}\right)$

$$
\begin{aligned}
& =\sum_{i=0}^{N} \mathscr{H}^{1}\left(\partial S^{*+} \cap \mathscr{L}\left(G_{i} \times(-a, a)\right)\right)+\sum_{i=1}^{N} \mathscr{H}^{1}\left(\partial S^{*+} \cap \mathscr{L}\left(B_{i} \times(-a, a)\right)\right) \\
& \leq \sum_{i=0}^{N}\left(\mathscr{H}^{1}\left(\left\{\mathscr{L}\left(q, f_{+}(q)\right): q \in G_{i}\right\}\right)+\sum_{j \in \mathbb{N}}\left|\widetilde{J}_{i, j}\right|\right)+\sum_{i=1}^{N}\left(\left|f_{+}^{l}\left(q_{i}\right)-f_{+}^{r}\left(q_{i}\right)\right|+\frac{\varepsilon}{N}\right) \\
& \leq \mathscr{H}^{1}\left(\left\{\mathscr{L}\left(q, f_{+}(q)\right): q \in \bigcup_{i=0}^{N} G_{i}\right\}\right)+\sum_{i>N}\left|J_{i}\right|+\sum_{i=1}^{N}\left(\left|f_{+}\left(q_{i}^{l}\right)-f_{+}\left(q_{i}^{r}\right)\right|+2 \frac{\varepsilon}{N}\right) \\
& \leq \mathscr{H}^{1}\left(\left\{\mathscr{L}\left(q, f_{+}(q)\right): q \in \bigcup_{i=0}^{N} G_{i}\right\}\right)+\varepsilon+\sum_{i=1}^{N}\left(\mathscr{H}^{1}\left(\gamma_{i}\right)+2 \frac{\varepsilon}{N}\right) \\
& \leq \mathscr{H}^{1}\left(\partial S^{+}\right)+3 \varepsilon .
\end{aligned}
$$


Since $\varepsilon>0$ was arbitrary, this step is concluded.

Step VI. Conclusion. By (10), we know that

$$
\partial S \supseteq \partial S^{+} \cup \partial S^{-} \cup \partial S^{l} \cup \partial S^{r},
$$

and the union is disjoint. But, as noticed at the end of Step II, we have

$$
\partial S^{*}=\partial S^{*+} \cup \partial S^{*-} \cup \partial S^{* l} \cup \partial S^{* r} .
$$

By Step V we know that $\mathscr{H}^{1}\left(\partial S^{+}\right) \geq \mathscr{H}^{1}\left(\partial S^{*+}\right)$, and, in the same way, we know that $\mathscr{H}^{1}\left(\partial S^{-}\right) \geq \mathscr{H}^{1}\left(\partial S^{*-}\right)$. Let us then focus for a moment on $\partial S^{l}$ and on $\partial S^{* l}$. If $\Gamma_{S}$ is compact, they are both empty. Otherwise, $\partial S^{l}$ is a curve between $\mathscr{L}\left(l, f_{-}(l)\right)$ and $\mathscr{L}\left(l, f_{+}(l)\right)$, while $\partial S^{* l}$ is the segment joining the same points. As a consequence, one has $\mathscr{H}^{1}\left(\partial S^{l}\right) \geq \mathscr{H}^{1}\left(\partial S^{* l}\right)$, and, similarly, $\mathscr{H}^{1}\left(\partial S^{r}\right) \geq \mathscr{H}^{1}\left(\partial S^{* r}\right)$. Adding up the four inequalities, we finally get that $\mathscr{H}^{1}(\partial S) \geq \mathscr{H}^{1}\left(\partial S^{*}\right)$.

Concerning formula (8), it is immediate to obtain for smooth functions $f_{-}$and $f_{+}$, while the generalization for $B V$ functions is standard.

With the above result at hand, it will be quite easy to obtain the lower bound.

Lemma 2.4 (lower bound). For a curved strip $\Omega_{\Gamma, a}$ of any kind, one has

$$
h\left(\Omega_{\Gamma, a}\right) \geq \frac{1}{a} .
$$

Moreover, if the inequality above is an equality and there is a Cheeger set, then this Cheeger set must be $\Omega_{\Gamma, a}$ itself.

Proof. Let $S$ be any open connected set of finite perimeter in $\Omega_{\Gamma, a}$, and let $S^{*}$ be as in Definition 2.2. Setting

$$
\begin{aligned}
& t_{-}:=\inf \left\{f_{-}(q): q \in \Gamma_{S}\right\}, \\
& t_{+}:=\sup \left\{f_{+}(q): q \in \Gamma_{S}\right\},
\end{aligned}
$$

we can easily estimate

$$
\begin{aligned}
\left|S^{*}\right| & =\int_{\Gamma} \int_{f_{-}(q)}^{f_{+}(q)}(1-\kappa(q) t) d t d q \\
& =\int_{\Gamma}\left(f_{+}(q)-f_{-}(q)\right)\left(1-\kappa(q) \frac{f_{+}(q)+f_{-}(q)}{2}\right) d q \\
& \leq\left(t_{+}-t_{-}\right) \int_{\Gamma}\left(1-\kappa(q) \frac{f_{+}(q)+f_{-}(q)}{2}\right) d q .
\end{aligned}
$$


On the other hand, by (8) it is easy to estimate the perimeter of $S^{*}$ as

$$
\begin{aligned}
P\left(S^{*}\right)= & \int_{\Gamma_{S}} \sqrt{\left(1-\kappa(q) f_{+}(q)\right)^{2}+f_{+}^{\prime}(q)^{2}} d q \\
& +\int_{\Gamma_{S}} \sqrt{\left(1-\kappa(q) f_{-}(q)\right)^{2}+f_{-}^{\prime}(q)^{2}} d q+\left|D_{S} f_{+}\right|\left(\Gamma_{S}\right) \\
& +\left|D_{S} f_{-}\right|\left(\Gamma_{S}\right)+\left(f_{+}\left(q_{0}\right)-f_{-}\left(q_{0}\right)\right)+\left(f_{+}\left(q_{1}\right)-f_{-}\left(q_{1}\right)\right) \\
\geq 2 & \int_{\Gamma_{S}}\left(1-\kappa(q) \frac{f_{+}(q)+f_{-}(q)}{2}\right) d q
\end{aligned}
$$

simply by neglecting both the absolutely continuous and the singular part of $D f$. Hence, thanks to Lemma 2.3, we can readily deduce that

$$
\frac{P(S)}{|S|} \geq \frac{P\left(S^{*}\right)}{\left|S^{*}\right|} \geq \frac{2}{t_{+}-t_{-}} \geq \frac{1}{a},
$$

where the last inequality is due to the trivial bounds $-a \leq t_{-}<t_{+} \leq a$. Finally, if $h\left(\Omega_{\Gamma, a}\right)=1 / a$ and there is some Cheeger set $\mathscr{C}=\mathscr{C}_{\Omega_{\Gamma, a}}$, then all the preceding inequalities must be equalities for $S=\mathscr{C}$, from which it immediately follows that $f_{+}$and $f_{-}$are constant, and that $t_{ \pm}= \pm a$. Thus $\mathscr{C}=\Omega_{\Gamma, a}$.

Remark 2.5. As a consequence of ( 3 ) for $p=2$, from the above result we get the lower bound

$$
\lambda_{2}\left(\Omega_{\Gamma, a}\right) \geq \frac{1}{4 a^{2}},
$$

which is in fact weaker than the bound

$$
\lambda_{2}\left(\Omega_{\Gamma, a}\right) \geq \frac{j_{0,1}^{2}}{4 a^{2}}
$$

known from [Exner et al. 2004]. Here, $j_{0,1} \approx 2.4$ denotes the first positive zero of the Bessel function $J_{0}$. In fact, an even better bound, reflecting the local geometry of $\Gamma$ and valid in arbitrary dimensions, is established in [Exner et al. 2004].

Remark 2.6. It is possible to establish the lower bound of Lemma 2.4 directly from Theorem 1.3 without using the "stripization" procedure $S^{*}$ of Definition 2.2 and its properties stated in Lemma 2.3. Inspired by the formula of [Bellettini et al. 2002, Sec. 11, Ex. 4] for the annulus, we introduce the function $V_{t}: \Gamma \times(-a, a) \rightarrow \mathbb{R}$,

$$
V_{t}(q, t):= \begin{cases}\frac{(1-\kappa(q) a)(1+\kappa(q) a)-(1-\kappa(q) t)^{2}}{2 a \kappa(q)(1-\kappa(q) t)} & \text { if } \kappa(q) \neq 0, \\ \frac{t}{a} & \text { if } \kappa(q)=0 .\end{cases}
$$

The value for vanishing curvature corresponds to taking the limit $\kappa(q) \rightarrow 0$ in the formula for positive curvatures. We check that, where the components are considered with respect to the coordinates $(q, t)$, the vector field $V(q, t):=\left(0, V_{t}(q, t)\right)$ 
satisfies $\|V\|_{L^{\infty}(\Gamma \times(-a, a))}=1$ and

$$
(\operatorname{div} V)(q, t)=\frac{1}{1-\kappa(q) t} \partial_{t}\left[(1-\kappa(q) t) V_{t}(q, t)\right]=\frac{1}{a}
$$

for every $(q, t) \in \Gamma \times(-a, a)$. Hence the searched lower bound is a consequence of Theorem 1.3. However, Lemma 2.3 is needed to establish some finer properties of the Cheeger constant and Cheeger set.

\section{The main results}

This section is devoted to show our two main results, namely Theorem 3.1, which deals with the case of curved annuli or non-finite curved strips, and Theorem 3.2, which deals with finite curved strips.

\section{The case of a curved annulus and that of a non-finite curved strip.}

Theorem 3.1. Let $\Gamma$ be compact, infinite, or semi-infinite. Then

$$
h\left(\Omega_{\Gamma, a}\right)=\frac{1}{a} .
$$

In particular:

(i) If $\Gamma$ is compact (that is, $\Omega_{\Gamma, a}$ is a curved annulus), then the infimum of (1) is attained and the unique Cheeger set is $\mathscr{b}_{\Omega_{\Gamma, a}}=\Omega_{\Gamma, a}$.

(ii) If $\Gamma$ is infinite or semi-infinite (that is, $\Omega_{\Gamma, a}$ is an infinite or semi-infinite curved strip), then the infimum of (1) is not attained, but the sequence $\Omega_{\Gamma_{L}, a}$ of Lemma 2.1 is an optimizing sequence for $L \rightarrow \infty$.

Proof. The equality (15) follows directly from the upper estimate of Lemma 2.1 and the lower estimate of Lemma 2.4.

From the characterization of Lemma 2.4, moreover, we know that the unique possible Cheeger set is the whole $\Omega_{\Gamma, a}$. Since this set has an infinite area and perimeter in the case of an infinite or semi-infinite curved strip, we get the nonexistence result of a minimizer for the case (ii), while the fact that $\Omega_{\Gamma_{L}, a}$ is a minimizing sequence for $L \rightarrow \infty$ follows by Lemma 2.1. On the other hand, in case (i) we know by compactness that some Cheeger set must exist, hence the existence and uniqueness of the whole $\Omega_{\Gamma, a}$ as a Cheeger set again comes by Lemma 2.4.

\section{The case of a finite curved strip.}

Theorem 3.2. Let $\Gamma$ be noncomplete and finite (hence, $\Omega_{\Gamma, a}$ is a finite curved strip). Then there exists a positive universal constant $c$ such that

$$
\frac{1}{a}+\frac{c}{|\Gamma|} \leq h\left(\Omega_{\Gamma, a}\right) \leq \frac{1}{a}+\frac{2}{|\Gamma|} .
$$


For instance, one may take $c=\frac{1}{400}$. Moreover, the infimum in (1) is attained for some connected set $\mathscr{C}_{\Omega_{\Gamma, a}} \subsetneq \Omega_{\Gamma, a}$.

Proof. Concerning the existence of a Cheeger set $\mathscr{C}=\mathscr{C}_{\Omega_{\Gamma, a}}$, and in particular of a connected one, this follows by Theorem 1.1. From the same Theorem, we know also that $\partial \mathscr{C} \cap \Omega_{\Gamma, a}$ is made by arcs of circle of radius $h\left(\Omega_{\Gamma, a}\right)^{-1}$, and, again by Theorem 1.1, it cannot coincide with the whole set $\Omega_{\Gamma, a}$, since $\mathscr{C}$ may not have corners. As a consequence, by the characterization of Lemma 2.4 we deduce that $h\left(\Omega_{\Gamma, a}\right)>1 / a$. To conclude, we have then only to give a proof of the bounds (16), which will be done in several steps.

Step I. The upper bound. Obtaining the upper bound is very easy: it is enough to recall that

$$
P\left(\Omega_{\Gamma, a}\right)=2|\Gamma|+4 a, \quad\left|\Omega_{\Gamma, a}\right|=2 a|\Gamma|,
$$

which was already checked, for instance in (7), and then

$$
h\left(\Omega_{\Gamma, a}\right) \leq \frac{P\left(\Omega_{\Gamma, a}\right)}{\left|\Omega_{\Gamma, a}\right|}=\frac{2|\Gamma|+4 a}{2 a|\Gamma|}=\frac{1}{a}+\frac{2}{|\Gamma|} .
$$

Step II. The lower bound: the behavior of the arcs of $\partial \mathscr{C} \cap \Omega_{\Gamma, a}$. Thanks to Theorem 1.1, we know that $\partial \mathscr{C}$ cannot have corners. Hence $\partial \mathscr{C} \cap \Omega_{\Gamma, a}$ is not empty, and it is done by some arcs of circle, all of radius $1 / h\left(\Omega_{\Gamma, a}\right)$ and hence strictly smaller than $a$ as noticed above, such that all four corners of $\Omega_{\Gamma, a}$ are ruled out from $\mathscr{C}$. Denoting by $q_{0}$ and $q_{1}$ the extreme points of $\Gamma$, let us call for simplicity "up", "down", "left", and "right" the four parts of $\partial \Omega_{\Gamma, a}$ given by the points of the form $\mathscr{L}(q, a), \mathscr{L}(q,-a), \mathscr{L}\left(q_{0}, t\right)$, and $\mathscr{L}\left(q_{1}, t\right)$ for $q \in \Gamma$ and $t \in(-a, a)$ respectively. We aim to show this:

Claim. All the arcs of circle of $\partial \mathscr{C} \cap \Omega_{\Gamma, a}$ connect two points of $\Omega_{\Gamma, a}$, at least one of which is either in the left or in the right part.

To show this claim, we have to exclude the case of an arc of circle starting and ending in the upper part, and the case of an arc connecting the up and the down (the case of an arc starting and ending in the bottom part is exactly the same as the first one).

Suppose first that there is an arc of circle connecting the points $P$ and $Q$, both in the upper part. Thus $P=\mathscr{L}\left(q^{\prime}, a\right)$ and $Q=\mathscr{L}\left(q^{\prime \prime}, a\right)$. By Theorem 1.1 , we know that the circle is tangent to $\partial \Omega_{\Gamma, a}$ at $P$ and $Q$, hence its center $O$ is the intersection between the two lines which are normal to $\partial \Omega_{\Gamma, a}$ at $P$ and $Q$, which are $t \mapsto \mathscr{L}\left(q^{\prime}, t\right)$ and $t \mapsto \mathscr{L}\left(q^{\prime \prime}, t\right)$. Since the radius $r$ of these circles is at most $a$, the two lines must intersect at the point

$$
\mathscr{L}\left(q^{\prime}, a-r\right) \equiv \mathscr{L}\left(q^{\prime \prime}, a-r\right),
$$

while this is impossible for any $r \leq 2 a$ because $\mathscr{L}$ is one-to-one. 
A very similar argument works assuming that an arc of circle connects the point $P=\mathscr{L}\left(q^{\prime}, a\right)$ in the upper part with the point $Q=\mathscr{L}\left(q^{\prime \prime},-a\right)$ in the lower part. Indeed, again the circle would be tangent to $\partial \Omega_{\Gamma, a}$ at both $P$ and $Q$, so that its center would be at the intersection of the segments $t \mapsto \mathscr{L}\left(q^{\prime}, t\right)$ and $t \mapsto \mathscr{L}\left(q^{\prime \prime}, t\right)$. This is impossible if the circle has radius smaller than $2 a$ for $q^{\prime} \neq q^{\prime \prime}$, but it is also impossible for a radius strictly smaller than $a$ in the case $q^{\prime}=q^{\prime \prime}$. This shows the Claim.

Notice now that by definition the left and the right parts of $\partial \Omega_{\Gamma, a}$ are segments, so the case of a circle starting and ending on the left is impossible, as is an arc starting and ending on the right. In conclusion, we now know that there can be either 2,3 , or 4 arcs of circle in $\partial \mathscr{C}$. The simplest case is when there are four arcs, each of which make a "rounded corner". This happens, for instance, for a rectangle (that is, if $\Gamma$ is a segment), and more generally if $a$ is sufficiently small with respect to $|\Gamma|$. However, it is also possible that there are only three arcs, one of which connects the left and the right part of the boundary. This happens, for instance, whenever the upper or lower part of the boundary is very short due to a big (but still admissible) curvature of $\Gamma$. An example of this situation is a sector of an annulus with very small inner radius, which then is very similar to a triangle: in this case the boundary of $\mathscr{b}$ does not touch the inner circle (some examples of this kind are shown in the next section). As to the last possibility (only two arcs of circle both connecting left and right), we have no example in mind and it may be impossible, but we do not need to exclude this case within this proof. Indeed, in Steps III and IV we will show the theorem in the case of four rounded corners, while in Step V we will show how it is always possible to reduce to this case.

Step III. The lower bound: the case when $\mathscr{b}$ has four rounded corners; statement of the Claim (18). To show the lower bound, we start from the case when $\mathscr{C}$ has four rounded corners. Let us recall that, as shown by (8) in Lemma 2.3, one has

$$
\begin{aligned}
P(\mathfrak{b})=\int_{\Gamma} & \sqrt{\left(1-\kappa(q) f_{+}(q)\right)^{2}+f_{+}^{\prime}(q)^{2}} d q \\
& +\int_{\Gamma} \sqrt{\left(1-\kappa(q) f_{-}(q)\right)^{2}+f_{-}^{\prime}(q)^{2}} d q+\left|D_{s} f_{+}\right|(\Gamma) \\
& +\left|D_{s} f_{-}\right|(\Gamma)+\left(f_{+}\left(q_{0}\right)-f_{-}\left(q_{0}\right)\right)+\left(f_{+}\left(q_{1}\right)-f_{-}\left(q_{1}\right)\right),
\end{aligned}
$$

where $f_{ \pm}^{\prime} d q$ is the absolute continuous part of $D f_{ \pm}$and $D_{s} f_{ \pm}$its singular part (notice that, in the language of Definition 2.2, we have $\Gamma_{\mathscr{C}}=\Gamma$ thanks to Step II). In particular,

$$
P(\mathscr{C}) \geq \int_{\Gamma}\left(2-\kappa(q)\left(f_{+}(q)+f_{-}(q)\right)\right) d q
$$


We claim that, at least in the case when $\mathscr{C}$ has four corners,

$$
P(\mathscr{C}) \geq \int_{\Gamma}\left(2-\kappa(q)\left(f_{+}(q)+f_{-}(q)\right)\right) d q+\frac{1}{50} a .
$$

We will prove this estimate in the next step. Now we show how this implies the thesis. We can easily estimate the area of $\mathscr{C}$ as in (14):

$$
\begin{aligned}
|\mathscr{C}| & =\int_{\Gamma} \int_{f_{-}(q)}^{f_{+}(q)}(1-\kappa(q) t) d t d q \\
& =\int_{\Gamma}\left(f_{+}(q)-f_{-}(q)\right)\left(1-\kappa(q) \frac{f_{+}(q)+f_{-}(q)}{2}\right) d q \\
& \leq a \int_{\Gamma}\left(2-\kappa(q)\left(f_{+}(q)+f_{-}(q)\right)\right) d q .
\end{aligned}
$$

Hence, using (18), we get (16) because, recalling that $a\|\kappa\|_{L^{\infty}(\Gamma)} \leq 1$ (as pointed out in Section 1),

$$
\begin{aligned}
h\left(\Omega_{\Gamma, a}\right) & =\frac{P(\mathscr{C})}{|\mathscr{C}|} \geq \frac{\int_{\Gamma}\left(2-\kappa(q)\left(f_{+}(q)+f_{-}(q)\right)\right) d q+\frac{1}{50} a}{a \int_{\Gamma}\left(2-\kappa(q)\left(f_{+}(q)+f_{-}(q)\right)\right) d q} \\
& =\frac{1}{a}+\frac{1}{50 \int_{\Gamma}\left(2-\kappa(q)\left(f_{+}(q)+f_{-}(q)\right)\right) d q} \\
& \geq \frac{1}{a}+\frac{1}{50|\Gamma|\left(2+2 a\|\kappa\|_{L^{\infty}(\Gamma)}\right)} \geq \frac{1}{a}+\frac{1}{200|\Gamma|} .
\end{aligned}
$$

Step IV. The lower bound: the case when $\mathscr{C}$ has four rounded corners; proof of Claim (18). We show that, assuming that $\partial \mathscr{C} \cap \Omega_{\Gamma, a}$ consists of four arcs of circle, (18) holds. This will be done by considering a single arc. To choose it, we start by noticing that (17) already trivially implies (18) if

$$
f_{+}\left(q_{1}\right)-f_{-}\left(q_{1}\right) \geq \frac{1}{50} a .
$$

As a consequence, we can assume that

$$
f_{+}\left(q_{1}\right) \leq \frac{1}{100} a,
$$

and we concentrate on the arc of circle corresponding to the "upper right corner". Of course, if (20) were not true, one could assume $f_{-}\left(q_{1}\right) \geq-a / 100$ and then make the completely symmetric considerations on the "lower right corner". As shown in Figure 4, we call the arc of circle that we are considering $\gamma$, and we can also look at $\gamma$ in the reference rectangle, where of course it is no longer part of a circle. We define the length $\eta$ as in Figure 4. 


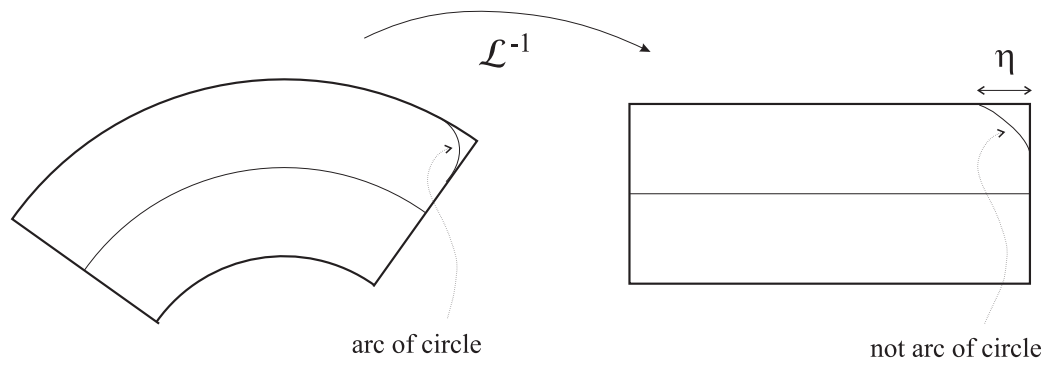

Figure 4. The situation (both in $\Omega$ and in the reference configuration) of Step IV.

We denote by $\Gamma_{\gamma}$ the part of $\Gamma$ related to the curve $\gamma . \Gamma_{\gamma}$ is therefore the subset of $\Gamma$ such that

$$
\gamma=\left\{\left(q, f_{+}(q)\right): q \in \Gamma_{\gamma}\right\},
$$

we can subdivide $\Gamma_{\gamma}$ into two parts, namely

$$
\Gamma_{1}:=\left\{q \in \Gamma_{\gamma}:\left|f_{+}^{\prime}(q)\right|<\frac{1}{5}\right\}, \quad \Gamma_{2}:=\left\{q \in \Gamma_{\gamma}:\left|f_{+}^{\prime}(q)\right| \geq \frac{1}{5}\right\} .
$$

Notice that the above subdivision makes sense because $f_{+}$has no singular part inside $\Gamma_{\gamma}$ (since the image of its graph under $\mathscr{L}$ is an arc of circle). By definition,

$$
\int_{\Gamma_{1}}\left|f_{+}^{\prime}(q)\right| d q \leq \frac{1}{5}\left|\Gamma_{1}\right| \leq \frac{1}{5} \eta \leq \frac{2}{5} a .
$$

In the last inequality we used the fact that $\eta \leq 2 a$, which is true because $\gamma$ is an arc of circle or radius smaller than $a$ (keep in mind that by Lemma 2.4 we already know that $\left.h\left(\Omega_{\Gamma, a}\right) \geq 1 / a\right)$ and the lengths in the reference rectangle are at most the double of the true lengths (recall also that by Theorem 1.1 the arcs of circle touch $\partial \Omega_{\Gamma, a}$ tangentially). But then, thanks to (20), one has

$$
\int_{\Gamma_{\gamma}}\left|f_{+}^{\prime}(q)\right| d q \geq \frac{99}{100} a
$$

so that by $(21)$ we get

$$
\int_{\Gamma_{2}}\left|f_{+}^{\prime}(q)\right| d q \geq\left(\frac{99}{100}-\frac{2}{5}\right) a=\frac{59}{100} a .
$$

Recalling again that $0<1-\kappa(q) f_{+}(q)<2$, a trivial calculation ensures that for any $q \in \Gamma_{2}$

$$
\sqrt{\left(1-\kappa(q) f_{+}(q)\right)^{2}+f_{+}^{\prime}(q)^{2}} \geq\left(1-\kappa(q) f_{+}(q)\right)+\frac{1}{25}\left|f_{+}^{\prime}(q)\right| .
$$


Hence, thanks to (22) and (23), we can estimate the length of $\gamma$ as

$$
\begin{aligned}
|\gamma| & =\int_{\Gamma_{\gamma}} \sqrt{\left(1-\kappa(q) f_{+}(q)\right)^{2}+f_{+}^{\prime}(q)^{2}} d q \\
& \geq \int_{\Gamma_{\gamma}}\left(1-\kappa(q) f_{+}(q)\right) d q+\frac{1}{25} \int_{\Gamma_{2}}\left|f_{+}^{\prime}(q)\right| d q \\
& \geq \int_{\Gamma_{\gamma}}\left(1-\kappa(q) f_{+}(q)\right) d q+\frac{59}{25 \cdot 100} a .
\end{aligned}
$$

Recalling formula (17) for the perimeter of $\mathscr{b}$, we finally conclude that

$$
P(\mathscr{C}) \geq \int_{\Gamma}\left(2-\kappa(q)\left(f_{+}(q)+f_{-}(q)\right)\right) d q+\frac{59}{25 \cdot 100} a,
$$

thus finally getting (18).

Step V. The lower bound: general case. In this last step we conclude the proof of the theorem. Thanks to the above steps, we already know that the result holds in the case of four rounded corners, so we can now assume that $\partial^{\mathscr{C}}$ has only two or three arcs. In this case, there exist two maximal numbers $a^{ \pm} \leq a$ such that $\mathscr{b} \subseteq \mathscr{L}\left(\Gamma \times\left(-a^{-}, a^{+}\right)\right)$. Let us now introduce a new strip $\Omega_{\widetilde{\Gamma}, \tilde{a}}$ :

$$
\bar{t}:=\frac{a^{+}-a^{-}}{2}, \quad \widetilde{\Gamma}:=\mathscr{L}(\Gamma \times\{\bar{t}\}), \quad \tilde{a}:=\frac{a^{+}+a^{-}}{2} .
$$

Notice that there is a bijective map $\varphi: \Gamma \rightarrow \widetilde{\Gamma}$ given by $\varphi(q)=\mathscr{L}(q, \bar{t})$, and that since $\widetilde{\Gamma}$ is parallel to $\Gamma$ by construction, the normal vector $N(q)$ to $\Gamma$ at $q$ coincides with the normal vector $\widetilde{N}(\varphi(q))$ to $\widetilde{\Gamma}$ at $\varphi(q)$. Thus, $\Omega_{\widetilde{\Gamma}, \tilde{a}}$ being a subset of $\Omega_{\Gamma, a}$, the injectivity condition (5) trivially holds for $\widetilde{\Gamma}$ and $\tilde{a}$, and we can conclude that the strip $\Omega_{\widetilde{\Gamma}, \tilde{a}}$ is admissible for our purposes.

By construction, we have $\mathscr{C} \subseteq \Omega_{\widetilde{\Gamma}, \tilde{a}}$, so $\mathscr{C}$ is also the Cheeger set of $\Omega_{\widetilde{\Gamma}, \tilde{a}}$. Moreover, by maximality of $a^{ \pm}$we know that $\mathscr{C}$ touches all four parts of the boundary of $\Omega_{\widetilde{\Gamma}, \tilde{a}}$, so the preceding steps, and in particular (19), allow us to deduce that

$$
h\left(\Omega_{\Gamma, a}\right)=\frac{P(\mathscr{C})}{|\mathscr{C}|}=h\left(\Omega_{\widetilde{\Gamma}, \tilde{a}}\right) \geq \frac{1}{\tilde{a}}+\frac{1}{200|\widetilde{\Gamma}|} .
$$

Finally, by definition $\tilde{a} \leq a$, while

$$
|\widetilde{\Gamma}|=\int_{\Gamma}(1-\bar{t} \kappa(q)) d q \leq 2|\Gamma| .
$$

Thus, we get (16) with the constant $c=\frac{1}{400}$. 

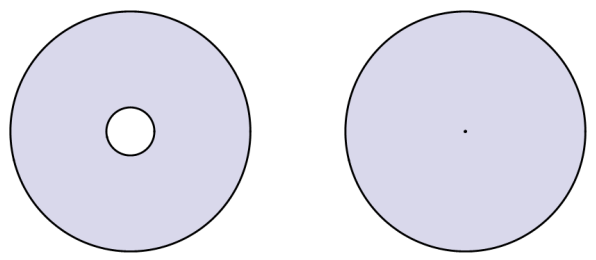

Figure 5. The annulus and the disc considered as its limit case.

\section{Solvable models}

In this section we discuss our results on the basis of several examples of curved strips about circles and circular arcs. They are referred to as solvable models since the determination of the Cheeger constant and the Cheeger set is reduced to solving an explicit algebraic equation. Where the exact solution is not available, we have solved the problem with the help of standard numerical tools.

Annuli. Probably the simplest example is given by annuli, that is, strips built about (full) circles; see Figure 5. Then the Cheeger set is the strip itself and the Cheeger constant equals half of the distance between the boundary curves. It follows from Theorem 3.1 that exactly the same situation holds for general curved annuli. Let us remark that discs can also be thought of as examples of curved strips. Indeed, a disc with its central point removed has the same Cheeger set (up to the point) and Cheeger constant as the disc, and the former set can be considered as the limit case of the annulus built about the circle of radius $a+\varepsilon$ when $\varepsilon \rightarrow 0+$.

Rectangles. The rectangle $\mathscr{R}_{a, b}:=(-b, b) \times(-a, a)$, with $a, b>0$, can be considered as a strip built about the segment $\Gamma:=(-b, b) \times\{0\}$. Using Theorem 1.2, it is easy to find its Cheeger constant explicitly:

$$
h\left(\mathscr{R}_{a, b}\right)=\frac{a+b+\sqrt{(a-b)^{2}+\pi a b}}{2 a b} .
$$

Notice the scaling $h\left(\mathscr{R}_{a, b}\right)=h\left(\mathscr{R}_{1, b / a}\right)$. The procedure also determines the Cheeger set of $\mathscr{R}_{a, b}$ as the rectangle with its corners rounded off by circular arcs of radius $h(\mathscr{R})^{-1}$; see Figure 6.

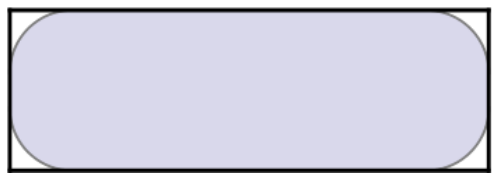

Figure 6. The rectangle and its Cheeger set (light gray) for $b / a=3$. 

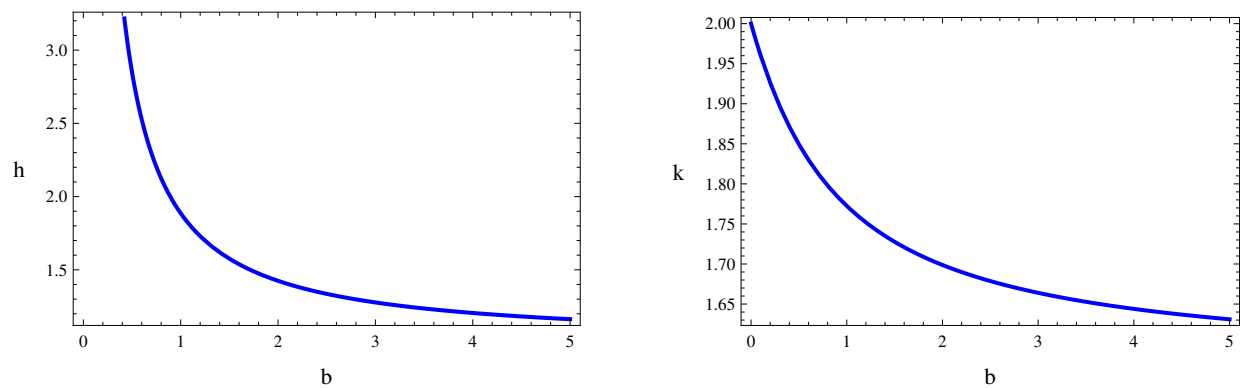

Figure 7. The Cheeger constant $h$ and the quantity $k$ for rectangles with $a=1$.

The Cheeger constant can be written as

$$
h\left(\mathscr{R}_{a, b}\right)=\frac{1}{a}+\frac{k(a, b)}{|\Gamma|}, \quad \text { where } k(a, b):=\frac{a-b+\sqrt{(a-b)^{2}+\pi a b}}{a}
$$

and $|\Gamma|=2 b$. Notice the scaling $k(a, b)=k(1, b / a)$. It is straightforward to check that $b / a \mapsto k(a, b)$ is a decreasing function with the limits $k(a, b) \rightarrow 2$ as $b / a \rightarrow 0$ and $k(a, b) \rightarrow \pi / 2$ as $b / a \rightarrow \infty$. Hence the upper bound of Theorem 3.2 becomes sharp in the limit of very narrow rectangles. The dependence of the Cheeger constant $h$ and of the quantity $k$ on rectangle parameters is shown in Figure 7.

Sectors. Let $\Gamma_{a}$ be the circle of curvature $\kappa=a^{-1}$ and consider its part $\Gamma_{a}^{\alpha}$ of length $\left|\Gamma_{a}^{\alpha}\right|=\alpha a$, with any $\alpha \in(0,2 \pi)$; see Figure 8. The corresponding strip $\Omega_{a}^{\alpha}:=\Omega_{\Gamma_{a}^{\alpha}, a}$ does not satisfy the assumption (5). However, since $\mathscr{L}$ is in fact injective in $\Gamma_{a} \times(-a, a)$, it can be considered as a limit case of admissible strips along corresponding parts of the circle of radius $a+\varepsilon$ when $\varepsilon \rightarrow 0+$.

The Cheeger constant and the Cheeger set of $\Omega_{a}^{\alpha}$ can be found as follows. First, we construct a family of domains $S_{r}$, with $r \in(0, a)$, defined by rounding off the corners in $\Omega_{a}^{\alpha}$ of angle smaller than $\pi$ by circular arcs of radius $r$. This can be done by a straightforward usage of elementary geometric rules. Secondly, we

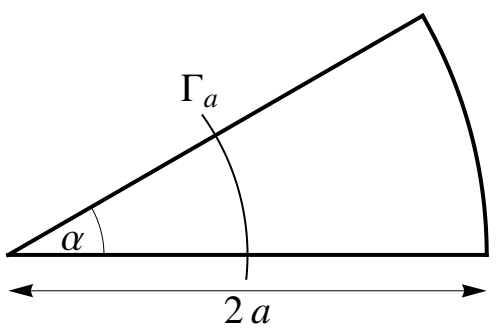

Figure 8. The sector of a disc considered as a strip built about the $\left(\frac{\alpha}{2 \pi}\right)$-th part of a circle. 

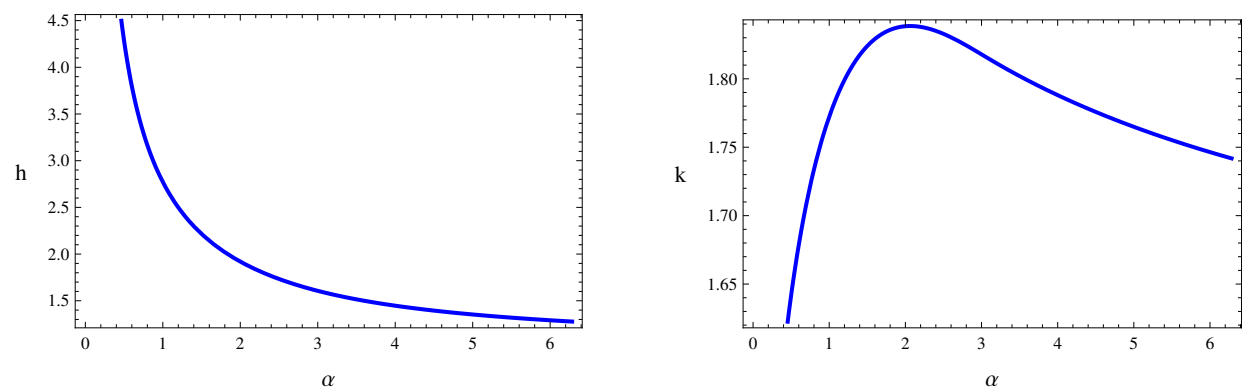

Figure 9. The Cheeger constant $h$ and the constant $k$ for sectors with $a=1$.

minimize the quotient $P\left(S_{r}\right) /\left|S_{r}\right|$ with respect to $r$, which is done with the help of a numerical optimization. The minimum of the quotient corresponds to the Cheeger constant, and the minimizer is the Cheeger set. The procedure is equivalent to using Theorem 1.2, which seems to remain valid also for $\alpha>\pi$, corresponding to nonconvex sectors.

In view of the obvious scaling $h\left(\Omega_{a}^{\alpha}\right)=h\left(\Omega_{1}^{\alpha}\right) / a$, one can set $a=1$, without loss of generality. The dependence of the Cheeger constant on $\alpha$ is shown in Figure 9. Table 1 contains numerical values for some specific angles.

Writing the Cheeger constant as

$$
h\left(\Omega_{a}^{\alpha}\right)=\frac{1}{a}+\frac{k(\alpha)}{\left|\Gamma_{a}^{\alpha}\right|},
$$

we also study the dependence of the constant $k(\alpha)$ on $\alpha$; see Figure 9 and Table 1. The third value of $\alpha$ in Table 1 corresponds to the maximal point of the curve $\alpha \mapsto k(\alpha)$ from Figure 9. In any case, we see that the upper bound of Theorem 3.2 is quite good for all the sectors. Finally, Figure 10 shows a numerical approximation of the Cheeger sets for some annuli.

\begin{tabular}{|c|ccccccc|}
\hline$\alpha$ & $\pi / 10$ & $\pi / 2$ & $0.656749 \pi$ & $3 \pi / 4$ & $\pi$ & $3 \pi / 2$ & $2 \pi$ \\
\hline$h\left(\Omega_{1}^{\alpha}\right)$ & 5.92687 & 2.16358 & 1.89111 & 1.77915 & 1.57714 & 1.37582 & 1.27722 \\
$k\left(\Omega_{1}^{\alpha}\right)$ & 1.54782 & 1.82774 & 1.83856 & 1.83583 & 1.81315 & 1.77101 & 1.74184 \\
\hline
\end{tabular}

Table 1. The Cheeger constant $h$ and the constant $k$ for sectors with $a=1$.

\section{Acknowledgements}

The authors acknowledge the hospitality of the Nuclear Physics Institute ASCR in Řež and the University of Pavia. 


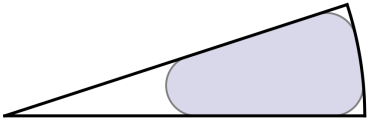

$\alpha=\pi / 10$

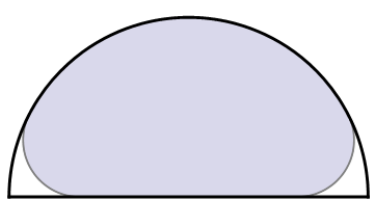

$\alpha=\pi$

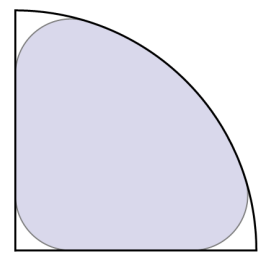

$\alpha=\pi / 2$

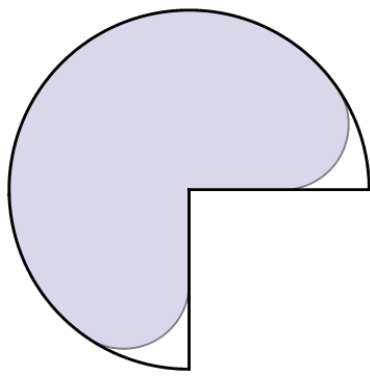

$\alpha=3 \pi / 2$

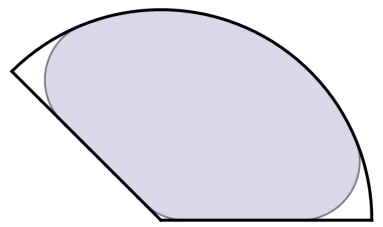

$\alpha=3 \pi / 4$

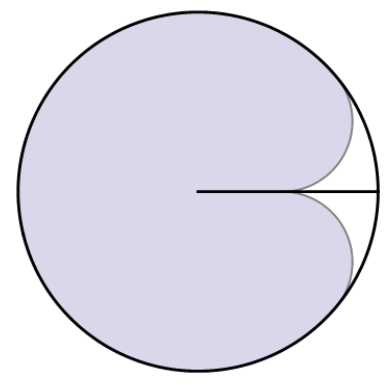

$\alpha=2 \pi$

Figure 10. The sectors and their Cheeger sets (light gray).

\section{References}

[Alter et al. 2005] F. Alter, V. Caselles, and A. Chambolle, "Evolution of characteristic functions of convex sets in the plane by the minimizing total variation flow", Interfaces Free Bound. 7:1 (2005), 29-53. MR 2006b:35154 Zbl 1084.49003

[Ambrosio et al. 2000] L. Ambrosio, N. Fusco, and D. Pallara, Functions of bounded variation and free discontinuity problems, Oxford University Press, New York, 2000. MR 2003a:49002 Zbl 0957.49001

[Bellettini et al. 2002] G. Bellettini, V. Caselles, and M. Novaga, "The total variation flow in $\mathbb{R}^{N}$ ", J. Differential Equations 184:2 (2002), 475-525. MR 2003g:35105 Zbl 1036.35099

[Caselles et al. 2007] V. Caselles, A. Chambolle, and M. Novaga, "Uniqueness of the Cheeger set of a convex body”, Pacific J. Math. 232:1 (2007), 77-90. MR 2008j:49012 Zbl 1221.35171

[Duclos and Exner 1995] P. Duclos and P. Exner, "Curvature-induced bound states in quantum waveguides in two and three dimensions", Rev. Math. Phys. 7:1 (1995), 73-102. MR 95m:81239 Zbl 0837.35037

[Evans and Gariepy 1992] L. C. Evans and R. F. Gariepy, Measure theory and fine properties of functions, CRC Press, Boca Raton, FL, 1992. MR 93f:28001 Zbl 0804.28001

[Exner et al. 2004] P. Exner, P. Freitas, and D. Krejčiřík, "A lower bound to the spectral threshold in curved tubes", Proc. R. Soc. Lond. Ser. A Math. Phys. Eng. Sci. 460:2052 (2004), 3457-3467. MR 2006d:81100 Zbl 02185441

[Grieser 2006] D. Grieser, "The first eigenvalue of the Laplacian, isoperimetric constants, and the max flow min cut theorem", Arch. Math. (Basel) 87:1 (2006), 75-85. MR 2007e:35213 Zbl 1105. 35062 
[Hebey and Saintier 2006] E. Hebey and N. Saintier, "Stability and perturbations of the domain for the first eigenvalue of the 1-Laplacian", Arch. Math. (Basel) 86:4 (2006), 340-351. MR 2007b: 35245 Zbl 1099.58012

[Kawohl and Fridman 2003] B. Kawohl and V. Fridman, "Isoperimetric estimates for the first eigenvalue of the $p$-Laplace operator and the Cheeger constant", Comment. Math. Univ. Carolin. 44:4 (2003), 659-667. MR 2005g:35053 Zbl 1105.35029

[Kawohl and Lachand-Robert 2006] B. Kawohl and T. Lachand-Robert, "Characterization of Cheeger sets for convex subsets of the plane”, Pacific J. Math. 225:1 (2006), 103-118. MR 2007e:52002 Zbl 1133.52002

[Krejčiřík and Kříž 2005] D. Krejčiřík and J. Kříž, "On the spectrum of curved planar waveguides", Publ. Res. Inst. Math. Sci. 41:3 (2005), 757-791. MR 2006k:35244 Zbl 1113.35143

[Saintier 2007] N. Saintier, "Shape derivative of the first eigenvalue of the 1-Laplacian", preprint, 2007. arXiv 0706.0873v1

Received November 15, 2010. Revised October 2, 2011.

DAVID KREJČIŘÍK

DePartment of TheORETICAL PHySics

NUCLEAR PHYSICS INSTITUTE ASCR

25068 ŘEŽ

CZECh Republic

krjcirik@ujf.cas.cz

\author{
Aldo PRATElli \\ DIPARTIMENTO DI MATEMATICA \\ UNIVERSITÀ DI PAVIA \\ VIA FERRATA, 1 \\ I-27100 PAVIA \\ ITALY \\ aldo.pratelli@unipv.it
}




\title{
PACIFIC JOURNAL OF MATHEMATICS
}

\author{
http://pacificmath.org \\ Founded in 1951 by \\ E. F. Beckenbach (1906-1982) and F. Wolf (1904-1989)
}

\section{EDITORS}

V. S. Varadarajan (Managing Editor)

Department of Mathematics

University of California

Los Angeles, CA 90095-1555

pacific@math.ucla.edu

Vyjayanthi Chari

Department of Mathematics

University of California

Riverside, CA 92521-0135

chari@math.ucr.edu

\section{Robert Finn}

Department of Mathematics Stanford University

Stanford, CA 94305-2125

finn@math.stanford.edu

Kefeng Liu

Department of Mathematics

University of California

Los Angeles, CA 90095-1555

liu@math.ucla.edu
Darren Long

Department of Mathematics

University of California

Santa Barbara, CA 93106-3080

long@math.ucsb.edu

Jiang-Hua Lu

Department of Mathematics

The University of Hong Kong

Pokfulam Rd., Hong Kong jhlu@maths.hku.hk

Alexander Merkurjev

Department of Mathematics

University of California

Los Angeles, CA 90095-1555

merkurev@math.ucla.edu
Sorin Popa

Department of Mathematics University of California

Los Angeles, CA 90095-1555 popa@math.ucla.edu

Jie Qing

Department of Mathematics

University of California

Santa Cruz, CA 95064

qing@cats.ucsc.edu

Jonathan Rogawski

Department of Mathematics

University of California

Los Angeles, CA 90095-1555

jonr@math.ucla.edu

\section{PRODUCTION}

pacific@math.berkeley.edu

\section{SUPPORTING INSTITUTIONS}

ACADEMIA SINICA, TAIPEI

CALIFORNIA INST. OF TECHNOLOGY INST. DE MATEMÁTICA PURA E APLICADA KEIO UNIVERSITY

MATH. SCIENCES RESEARCH INSTITUTE NEW MEXICO STATE UNIV.

OREGON STATE UNIV.

\author{
STANFORD UNIVERSITY \\ UNIV. OF BRITISH COLUMBIA \\ UNIV. OF CALIFORNIA, BERKELEY \\ UNIV. OF CALIFORNIA, DAVIS \\ UNIV. OF CALIFORNIA, LOS ANGELES \\ UNIV. OF CALIFORNIA, RIVERSIDE \\ UNIV. OF CALIFORNIA, SAN DIEGO \\ UNIV. OF CALIF., SANTA BARBARA
}

\author{
UNIV. OF CALIF., SANTA CRUZ \\ UNIV. OF MONTANA \\ UNIV. OF OREGON \\ UNIV. OF SOUTHERN CALIFORNIA \\ UNIV. OF UTAH \\ UNIV. OF WASHINGTON \\ WASHINGTON STATE UNIVERSITY
}

These supporting institutions contribute to the cost of publication of this Journal, but they are not owners or publishers and have no responsibility for its contents or policies.

See inside back cover or pacificmath.org for submission instructions.

The subscription price for 2011 is US \$420/year for the electronic version, and \$485/year for print and electronic.

Subscriptions, requests for back issues from the last three years and changes of subscribers address should be sent to Pacific Journal of Mathematics, P.O. Box 4163, Berkeley, CA 94704-0163, U.S.A. Prior back issues are obtainable from Periodicals Service Company, 11 Main Street, Germantown, NY 12526-5635. The Pacific Journal of Mathematics is indexed by Mathematical Reviews, Zentralblatt MATH, PASCAL CNRS Index, Referativnyi Zhurnal, Current Mathematical Publications and the Science Citation Index.

The Pacific Journal of Mathematics (ISSN 0030-8730) at the University of California, c/o Department of Mathematics, 969 Evans Hall, Berkeley, CA 94720-3840, is published monthly except July and August. Periodical rate postage paid at Berkeley, CA 94704, and additional mailing offices. POSTMASTER: send address changes to Pacific Journal of Mathematics, P.O. Box 4163, Berkeley, CA 94704-0163.

PJM peer review and production are managed by EditFLOW ${ }^{\mathrm{TM}}$ from Mathematical Sciences Publishers.

PUBLISHED BY PACIFIC JOURNAL OF MATHEMATICS

at the University of California, Berkeley 94720-3840

A NON-PROFIT CORPORATION

Typeset in LATEX

Copyright $(2011$ by Pacific Journal of Mathematics 


\section{PACIFIC JOURNAL OF MATHEMATICS}

Volume $254 \quad$ No. $2 \quad$ December 2011

Curvatures of spheres in Hilbert geometry

ALEXANDER BORISENKO and EUGENE OLIN

A formula equating open and closed Gromov-Witten invariants and its applications 275

to mirror symmetry

KWOKWAI CHAN

A note on $p$-harmonic $l$-forms on complete manifolds

LiAng-CHu CHANG and CHIUng-JUe ANNA SUNG

The Cheeger constant of curved strips

DAVID KREJČIŘíK and ALdo PRATELLI

Structure of solutions of 3D axisymmetric Navier-Stokes equations near maximal points

\section{ZHEN LEI and QI S. ZHANG}

Local comparison theorems for Kähler manifolds

GANG LIU

Structurable algebras of skew-rank 1 over the affine plane

SUSANNE PUMPLÜN

An analogue of Krein's theorem for semisimple Lie groups

SANJOY PUSTI

Une remarque de dynamique sur les variétés semi-abéliennes

GAËL RÉMOND

Fourier transforms of semisimple orbital integrals on the Lie algebra of $\mathrm{SL}_{2}$

LOREN SPICE

On noncompact $\tau$-quasi-Einstein metrics

LIN FENG WANG

Decomposition of de Rham complexes with smooth horizontal coefficients for semistable reductions

QIHONG XIE

A differentiable sphere theorem inspired by rigidity of minimal submanifolds 